\title{
A Continuous Time Quantum Monte Carlo as an Impurity Solver for Strongly Correlated System
}

\author{
R. K. Rai, R. B. Ray, G. C. Kaphle, and O. P. Niraula
}

Journal of Nepal Physical Society

Volume 7, No 3, 2021

(Special Issue: ANPA Conference, 2021)

ISSN: 2392-473X (Print), 2738-9537 (Online)

\section{Editors:}

Dr. Nabin Malakar (Editor in chief)

Worcester State University

Dr. Pashupati Dhakal

Thomas Jefferson National Accelerator Facility, USA

Dr. Arjun Dahal

University of South Alabama, USA

Dr. Chiranjivi Lamsal

SUNY Platsburgh, USA

Dr. Dilli Raj Paudyal

University of Regina, Canada

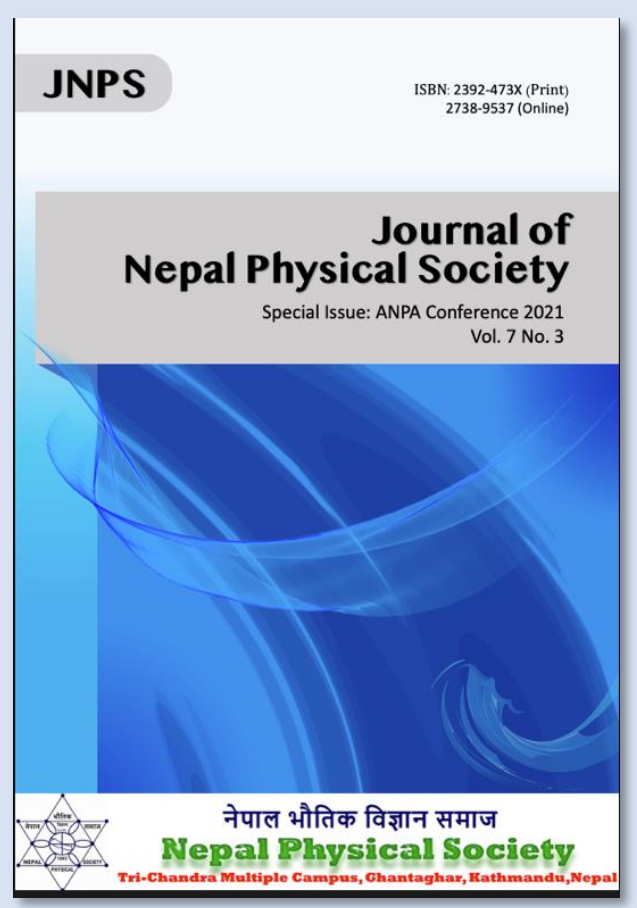

Managing Editor:

Dr. Binod Adhikari

St. Xavier's College, Kathmandu, Nepal

JNPS, 7 (3), 14-26 (2021)

DOI: http://doi.org/10.3126/jnphyssoc.v7i3.42185

Published by: Nepal Physical Society

P.O. Box: 2934

Tri-Chandra Campus

Kathmandu, Nepal

Email: npseditor@gmail.com 


\title{
A Continuous Time Quantum Monte Carlo as an Impurity Solver for Strongly Correlated System
}

\author{
R. K. Rai, ${ }^{1, \text { a) }}$ R. B. Ray, ${ }^{2}$ G. C. Kaphle, ${ }^{3, \text { b) }}$ and O. P. Niraula ${ }^{3}$ \\ 1) Patan Multiple Campus, Tribhuvan University, Patandhoka, Lalipur, Nepal \\ 2) Amrit Campus, Tribhuvan University, Lainchaur, Kathmandu, Nepal \\ ${ }^{3)}$ Central Department of Physics, Tribhuvan University, Kirtipur, Kathmandu, Nepal \\ ${ }^{a)}$ Corresponding Email: rairajk@gmail.com \\ b) Corresponding Email: gck223@gmail.com
}

\begin{abstract}
We assess the continuous-time quantum Monte Carlo (CT-QMC) technique with hybridization expansion for solving the electronic structure of the strongly correlated system $\mathrm{La}_{\mathrm{x}} \mathrm{Sr}_{1-\mathrm{x}} \mathrm{VO}_{3}$. The impurity solver method implemented here shows the fair agreement with the other available Monte Carlo techniques. From the study, it is found that the CT-QMC technique clearly distinguishes metallic phase, quasiparticle phase and insulating phases of the system depending upon the strength of the correlation. In case of $\mathrm{La}_{0.33} \mathrm{Sr}_{0.67} \mathrm{VO}_{3}$ system the metal-insulator transition is found to be at $\mathrm{U}=4.5 \mathrm{eV}$ for $\beta=6(\mathrm{eV})^{-1}$. The value of $\mathrm{U}$ depends with the value of $\beta$, and also the value of Hund's coupling $(\mathrm{J})$ and bandwidth $(\mathrm{W})$. This technique allows the particle to exchange with the reservoir of the particles and the impurity sites, which is accounted numerically to treat the temporal fluctuation of the fermionic systems termed as dynamical mean field theory (DMFT). This theory is used to explain the phenomena of MottHubbard metal insulator transition of the materials which are applicable for designing the Mottronics, Neuromorphic computing, Quantum computing and resistive memory devices.
\end{abstract}

Received: 14 August 2021; Revised: 30 October 2021; Accepted: 15 November 2021

Keywords: CT-QMC, DFT, DMFT, MIT, Neuromorphic Computing.

\section{INTRODUCTION}

The strongly correlated systems, having Mott-Hubbard metal-insulator transition (MIT), are of great interest because of their application in Mottronics, Neuromorphic computing, Quantum computing and volume resistive memory devices [1]. The properties of such materials are depending on tunable parameters like Coulomb interaction (U), thermodynamic parameter $(\beta)$, Hund's coupling (J), and bandwidth (W) [2]. In particular the MIT phenomena is explained through dynamical mean-field theory in addition to the density functional theory (DFT), which accounts the temporal fluctuations in many-body system at which spatial fluctuation is negligible [3]. Here, we describe CT-QMC technique with hybridization expansion as the impurity solver of DMFT equation to study MIT for strongly correlated system, $\mathrm{La}_{\mathrm{x}} \mathrm{Sr}_{1-\mathrm{x}} \mathrm{VO}_{3}$. The strongly correlated systems have incoherent electronic property, which is mainly due to the electron-electron interaction on the lattice sites. MIT mainly causes due to charge and spin degree of freedom, and orbital orderings. These are the parameters, which also explain the nature of Anderson localization, Jahn-Teller distortion and the unpredictable incoherent metallic behaviours near the Mott metal-insulator phase transition. The orbital degree of freedom essentially important to understand the nature of d-orbital ordering associated to behavior of MIT [2, 3, 4]. Controlling the parameters $\mathrm{U}, \mathrm{J}$ and $\beta$ through filling control, bandwidth control and dimensionality control can tune these properties. For this, one can adopt the DFT plus the DMFT approach as well as experimental approach [5]. Kim, M. et al. used DMFT combining with ab initio density functional methods with the generalized gradient approximation (GGA) to account the itinerancy and localization of electrons [6]. Streltsov et al. also used the DMFT to predict the Hubbard satellite, which splits the conduction band of a metal and hence explain the Mott insulator [7]. Rubtsov et al. developed CT-QMC algorithm to study the interactions and superexchange of fermions, which is successfully implemented in multi-band model on non-local spin-flip terms 
[8]. Dirks et al. implemented the CT-QMC to obtain the precise and unbiased imaginary time data and the corresponding physical observables are obtained through analytic continuation using Maximum entropy model [9]. Chatterjee et al. explain how amplitudes of Friedel oscillations and the screening charge decrease with increasing the interaction, which is finally ceases in the Mott insulator regime with finite residual screening charges using CT-QMC [10]. Similarly, this technique used to study the realistic calculation of strongly correlated materials having $\mathrm{d}$ - and $\mathrm{f}$ - electrons by using a set of SU(2)-symmetric Kanamori Hamiltonian by Parragh et al. [11]. Kowalski et al. developed CT-QMC hybridization algorithm to study the splitting of d-orbitals and local Coulomb interactions, which is extremely useful to explain the Mott insulating behavior [12]. Experimentally, the angle resolved photoemission spectroscopy (ARPES) was used to probe the presence of Hubbard bands [13]. Here, in this communication we review the theoretical background of the solution of DMFT equation implementing CT-QMC technique with hybridization expansion to explain the phenomena of Mott-Hubbard kinetics in superstructure of strongly correlated systems $\mathrm{La}_{\mathrm{x}} \mathrm{Sr}_{1-\mathrm{x}} \mathrm{VO}_{3}[14,15,16$, 17, 18, 19]. The process of implementation of reconstructing data from CT-QMC through maximum entropy model, which is useful to predict Mott phase transition of TMOs, has been discussed [20,21, 22, 23, 24, 25, 26, 27]. We believe present study helps to understand the properties of field driven MIT which is applicable for designing the Mottronics, Neuromorphic computing, Quantum computing, other resistive memory devices [28, $29,30,31,32,33,34]$.

\section{METHODOLOGY AND COMPUTATIONAL DETAILS}

We discuss the theoretical background of Monte Carlo technique, the kernel of the CT-QMC through which computer generates a series of pseudo random numbers. These pseudo random numbers are then used to either simulate naturally random processes, such as electronic fluctuation in lattice sites $[35,36]$. The explanations of theory related to CT-QMC for estimating MottHubbard MIT phase and implementation of technique to the $\mathrm{La}_{\mathrm{x}} \mathrm{Sr}_{1-\mathrm{x}} \mathrm{VO}_{3}$ has been discussed. Further computational detail and codes used for the generation of data were also be discussed. The Mott-Hubbard MIT in a solid, in fact arise due to quasi-particles behaviours of electrons, which can be characterized by (a) the presence or absence of spontaneously broken symmetry (b) the gapped or gapless low energy neutral particle excitations (c) the presence or absence of topological phase transitions [37]. The Mott transition problem of strongly correlated electronic system is directly addressed by the
DMFT framework for such materials [38]. The single site Hubbard Hamiltonian for representing an interacting system is,

$$
\mathrm{H}=-\mathrm{t} \sum_{\mathrm{ij}, \sigma} \mathrm{c}_{\mathrm{i} \sigma}^{\dagger} \mathrm{c}_{\mathrm{j} \sigma}+\mathrm{U} \sum_{\mathrm{i}} \mathrm{n}_{\mathrm{i} \uparrow} \mathrm{n}_{\mathrm{i} \downarrow},
$$

The non-local part of interaction is,

$$
\mathrm{H}_{\text {non-local }}=-\mathrm{t} \sum_{\mathrm{ij}, \sigma}\left(\mathrm{c}_{\mathrm{i} \sigma}^{\dagger} \mathrm{c}_{\mathrm{j} \sigma}+\mathrm{c}_{\mathrm{j} \sigma}^{\dagger} \mathrm{c}_{\mathrm{i} \sigma}\right)
$$

And the local part of interaction on site $i$ is

$$
\mathrm{H}_{\text {local }}=\mathrm{U} \sum_{\mathrm{i}} \mathrm{c}_{\mathrm{i} \uparrow}^{\dagger} \mathrm{c}_{\mathrm{i} \uparrow} \mathrm{c}_{\mathrm{i} \downarrow}^{\dagger} \mathrm{c}_{\mathrm{i} \downarrow}
$$

Considering the fermionic cases on site $\mathrm{i}$, we must realize that the hopping term does not conserve the particles number at the site. The single-site lattice model (Hubbard model) is then mapped onto a self-consistent quantum impurity model (Anderson impurity model) representing the interaction on real crystal lattice site $i$ as in Fig. 1. The impurity Hamiltonian is the basis of DMFT in which the various kinds of impurity solvers including CT-QMC method are implemented [39].

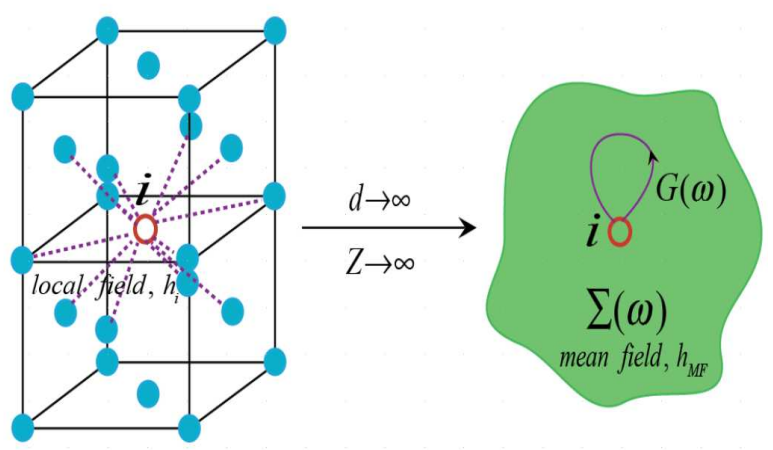

FIGURE 1. (Color online) The local field interaction for a fcc lattice. In the limit of infinite dimension $d \rightarrow \infty$ or $Z \rightarrow \infty$, the local field $h_{i}$ surrounding a single atom can be described by an effective field $h_{M F}$ with no spatial but only the fluctuations of the electronic system [40]

\section{The Anderson impurity model:}

The Anderson impurity Hamiltonian representing the many-body system is,

$$
\begin{gathered}
\mathscr{H}=\sum_{\sigma=\uparrow, \downarrow} \varepsilon_{0}(\mathrm{k}) \mathrm{a}_{\sigma}^{\dagger} \mathrm{a}_{\sigma}+\mathrm{Un}_{\mathrm{a} \uparrow \mathrm{n}_{\mathrm{a} \downarrow}} \\
+\sum_{\mathrm{k}, \sigma}\left[\mathrm{V}_{\mathrm{k}, \sigma}(\mathrm{k}) \mathrm{c}_{\mathrm{k}, \sigma}^{\dagger} \mathrm{c}_{\sigma}+\text { h.c. }\right] \\
+\sum_{\mathrm{k}, \sigma} \varepsilon_{\mathrm{k}, \sigma}(\mathrm{k}) \mathrm{c}_{\mathrm{k}, \sigma}^{\dagger} \mathrm{c}_{\mathrm{k}, \sigma}
\end{gathered}
$$


Where, $\varepsilon_{0}(k)$ is the energy level of the impurity, $a_{\sigma}^{\dagger}$ (corr. $a_{\sigma}$ ) is the creation (corr. annihilation) operator for an electron with spin $\sigma$ on the impurity, $\varepsilon_{k, \sigma}(k)$ is the energy spectrum of the bath $c_{\sigma}^{\dagger}$ (corr. $c_{\sigma}$ ) is the creation (corr. annihilation) operator for an electron with spin $\sigma$ and momentum $\mathrm{k}$ in the bath and $\mathrm{V}_{\mathrm{k}, \sigma}(\mathrm{k})$ is the hybridization parameter(coupling constant) [41]. The partition function associated with the impurity and its fermionic bath is,

$$
Z=\operatorname{Tr}[\exp -\beta \mathscr{H}]
$$

A very successful way to analyze the system using the numerical technique with the imaginary time formalism, the partition function is the path integral over Grassman variables [42] $a_{\sigma}^{\dagger}$ and $a_{\sigma}$ expressed as,

$$
\mathrm{Z}_{=}=\int \mathrm{D}\left[\mathrm{a}_{\sigma}^{\dagger}, \mathrm{a}_{\sigma}\right] \exp \left(-\mathrm{S}_{\mathrm{eff}}\right)
$$

Where, $S_{\text {eff }}$ is the effective action associated with the lattice Hubbard model Hamiltonian, which is solved by mapping onto a single-site impurity Anderson model Hamiltonian Eq. (4).

$$
\begin{gathered}
\mathrm{S}_{\mathrm{eff}}=-\sum_{\sigma} \int_{0}^{\beta} \mathrm{d} \tau \mathrm{d} \tau^{\prime} \mathrm{a}_{\sigma}^{\dagger}(\tau) \mathscr{G}_{0 \sigma}^{-1}\left(\tau-\tau^{\prime}\right) \mathrm{a}_{\sigma}\left(\tau^{\prime}\right) \\
+\int_{0}^{\beta} \mathrm{d} \tau \operatorname{Un}_{\mathrm{a} \uparrow}(\tau) \mathrm{n}_{\mathrm{a} \downarrow}(\tau)
\end{gathered}
$$

The non-interacting $(U=0)$ Green's function

$$
\mathscr{G}_{0 \sigma}^{-1}\left(i \omega_{v}\right)=i \omega_{v}-\varepsilon_{0}-\Delta_{\sigma}\left(i \omega_{v}\right)
$$

According to Matsubara, the hybridization part of interaction is,

$$
\Delta_{\sigma}\left(\mathrm{i} \omega_{v}\right)=\sum_{\mathrm{k}} \frac{\mid \mathrm{V}_{\left.\mathrm{k} \sigma(\mathrm{k})\right|^{2}}}{\mathrm{i} \omega_{v}-\varepsilon_{\mathrm{k} \sigma}(\mathrm{k})}
$$

Which describes the transition between the bath and the various orbitals [43].

\section{Evaluating the partition function with Monte Carlo sampling:}

The varieties of quantum Monte Carlo (QMC) methods are the most useful tools for the numerical study of manybody systems with strong correlated fermionic system [44]. Here, we are focusing our discussion on the CTQMC algorithm for fermions. This algorithm is a family of stochastic process for solving the Anderson impurity model at finite temperature. These methods consist of a
Hamiltonian involving a finite number of states and hybridization process, which allows particle exchange with the fermionic bath of these particles. They are important both in their own right and as a crucial ingredient in the dynamical mean field method of approximating the properties of interacting fermions on an infinite dimensional lattice sites [45]. The principle behind all of these algorithms is the same, which is stemming from earlier work on diagrammatic Monte Carlo [46]. In all cases, we need to implement the Monte Carlo Integration, in which the integral of a function can be converted to a discrete sum of the form,

$$
\int_{a}^{b} f(x) d x=\frac{b-a}{N} \sum_{i=1}^{N} f\left(a+i \frac{b-a}{N}\right)+O\left(\frac{1}{N}\right)
$$

There are other higher order numerical integrators such as Trapezoidal rule, Simpson rule etc. The Monte Carlo technique constitutes the sampling, errors, Markov chains and Metropolis-Hastings arguments for obtaining the result [47]. In fact, the Monte Carlo is a method computes the sums for a probability distribution, $\mathrm{p}(\mathrm{x})$ for a sampling function $\mathrm{f}(\mathrm{x})$ of the given configuration space $\mathrm{C}$ [40].

$$
\sum_{x} p(x) f(x)
$$

where, $p(x)>0$,

$$
\sum_{\mathrm{x}} \mathrm{p}(\mathrm{x})=1
$$

The variable, $\mathrm{x}=(\tau, \sigma, \ldots)$ represents the set of variables under study.

\section{Continuous time Partition Function Expansions in Configuration Space:}

The continuous-time partition function expansions for Monte-Carlo sampling with a typical infinite series on imaginary time parameters is,

$$
\mathrm{Z}=\sum_{\mathrm{n}=0}^{\infty} \iiint_{0}^{\beta} \mathrm{d} \tau_{1} . . \mathrm{d} \tau_{\mathrm{n}} \mathrm{p}\left(\tau_{1}, \tau_{2}, \ldots, \tau_{\mathrm{n}}\right)
$$

This is the partition function representing the sum of the expansion orders from zero to infinity over $\mathrm{n}$ slices of imaginary time steps $\tau_{1}, \ldots \tau_{n}$, which is integrated over the probability densities, $\mathrm{p}\left(\tau_{1}, \ldots \tau_{n}\right)$ from $\tau=0$ to $\beta$, imaginary time intervals [48]. We need to sample terms with the weight, which is associated with the infinite configurations $\mathrm{C}$ (moves in the phase space) of this integral, contributing to the partition function. As for examples, let us start to write down some of the lowest orders of the integral explicitly. The first order calculation of the partition function of single parameter is, 


$$
\mathrm{Z}_{1}=\int_{0}^{\beta} \mathrm{d} \tau_{1} \mathrm{p}\left(\tau_{1}\right)
$$

The integrand is described uniquely by a set of imaginary time $\left\{\left(\tau_{1}\right)\right\}$, so that we can sample Eq. (12) up to first order using Monte Carlo technique [49], which generates a uniformly distributed random numbers of imaginary time, $\tau_{1}$ in the interval $(0, \beta)$, we may write,

$$
\mathrm{Z}_{1}=\lim _{\mathrm{N} \rightarrow \infty} \frac{1}{\mathrm{~N}} \sum_{0}^{\beta} \mathrm{p}\left(\tau_{\mathrm{j}}\right)
$$

Using analogy, the second order calculation is obtained by the another set of imaginary time $\left\{\left(\tau_{1}, \tau_{2}\right)\right\}$ and generating uniformly distributed value pairs $\left(\tau_{1}, \tau_{2}\right)$ in the interval $(0, \beta)$, we may write,

$$
\mathrm{Z}_{1}=\lim _{\mathrm{N} \rightarrow \infty} \frac{1}{\mathrm{~N}} \sum_{0}^{\beta} \mathrm{p}\left(\tau_{\mathrm{j}}, \tau_{2}\right)
$$

We need to sample the integral up to some finite order n max (say) and then truncate it. For sampling, the various terms, we employ Metropolis' algorithm [50] to accept or reject the possible transitions. The number of Monte Carlo samples may have only the Monte Carlo error, which scales as $\frac{1}{\sqrt{N}}$ with the samples [51]].

Let us define all the possible orders of configuration space $\mathrm{C}$ as,

$\mathrm{C}=\left\{\{.\},.\left\{\tau_{1}\right\},\left\{\tau_{1}, \tau_{2}\right\}, \ldots,\left\{\tau_{1}, \ldots, \tau_{\mathrm{n}}\right\}\right\}$

Which is the set of continuous imaginary time variables $\tau_{j}$. We should consider that the configurations are timeordered, i.e. $\tau_{1}<\tau_{2}<\ldots<\tau_{n}$ Each configuration contributes some value to the whole partition function. To avoid the sign problems, the expansion coefficients above are taken to be positive [52] otherwise sampling will be difficult.

\section{Markov Chain Transition (move):}

We use the Markov chain Monte Carlo method for sampling the configurations $C$ that contributes to the value of the partition function $\mathrm{Z}$. Assuming, $\mathrm{x}_{0}$ be the initial configuration (or zeroth order configuration), we need to proceed with random walk from a present configuration, $\mathrm{x}$ to a new one $y\left(x_{j}\right)$ in phase space as,

$$
\mathrm{x}_{0} \rightarrow \mathrm{x}_{1} \rightarrow \mathrm{x}_{2} \rightarrow \ldots \rightarrow \mathrm{x}_{\mathrm{n}+1} \ldots
$$

We have implemented the diagrammatic Monte Carlo codes by raising of the order for updating the transition, $\mathrm{x}_{\mathrm{n}} \rightarrow \mathrm{x}_{\mathrm{n}+1}$ i.e. the insertion of an additional vertex $\left(\tau_{j}\right)$ (adding link), or, lowering of the order, i.e. removal of an additional vertex $\left(\tau_{1}\right)$ (removal of link), or a local change on the same site with the same order $\left(\tau_{j} \rightarrow \tau_{j}^{\prime}\right)$, like a spinflip or the change of a $\tau$ [53] as in Fig. 2. For summing up all the probabilities, the Markov chain must reach a stationary distribution. We must make sure that the system be thermalized as well as the successive points in the Markov chain are clearly correlated. The autocorrelation effects must be taken into account while computing the Monte Carlo statistical errors [45]. To create a Markov chain of diagrams (moves) by assuming any two "moves", we can purpose the following moves: (i) Insertion of a link (anti-link): we choose a random imaginary time and insert a vertex with a spin randomly up or down with the imaginary time step, $\tau_{i}$. (ii) Removal of a link: choosing a random vertex of imaginary time step $\tau_{i}$ and remove it.

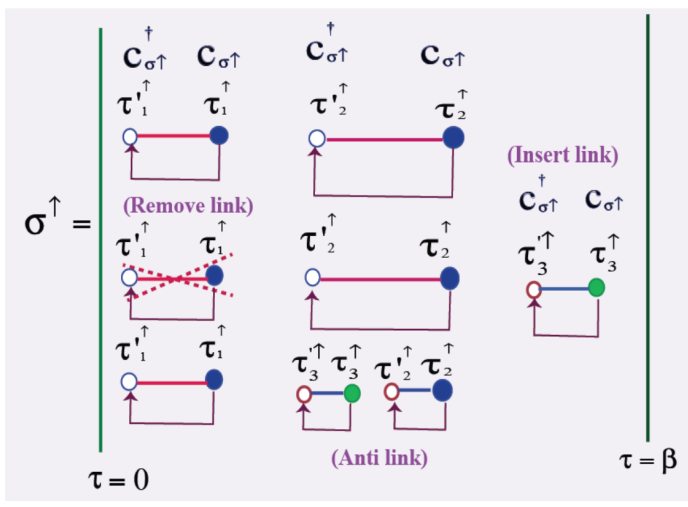

FIGURE 2. (Color online) Insertion and removal of vertices (interactions) update are illustrated in the above diagrams. The sampling process samples configurations according to their contribution to the partition function [54]

Starting from an arbitrary distribution of Markov chain process will converge exponentially to a stationary distribution $\mathrm{p}(\mathrm{x})$ if two the following two conditions are satisfied: (i) Ergodicity: any configuration $\mathrm{x}$ is reachable from any other configuration $y$ in a finite number of Markov steps. That is to say,

$\forall \mathrm{xy} \exists \mathrm{n}:\left(\mathrm{W}^{\mathrm{n}}\right)_{\mathrm{xy}} \neq 0$

(ii) Detailed balance: The probability $\mathrm{W}_{\mathrm{xy}}$ of transitioning from configuration $\mathrm{x}$ with probability density $\mathrm{p}(\mathrm{x})$ to configuration $\mathrm{y}$ with weight $\mathrm{p}(\mathrm{y})$ satisfies the detailed balance condition,

$$
\int_{\mathrm{c}} \mathrm{W}_{\mathrm{xy}} \mathrm{p}(\mathrm{x})=\mathrm{p}(\mathrm{y})
$$

This probability needs to fulfill the detailed balance condition to perform our updates using the Metropolis algorithm. Assuming that we have a configuration $x=\left\{\left(\tau_{1}\right), \ldots,\left(\tau_{n}\right)\right\}$ and try to insert a time vertex $\left(\tau_{n+1}\right)$ to obtain a configuration $y=\left\{\left(\tau_{1}\right), \ldots,\left(\tau_{n}\right),\left(\tau_{n+1}\right)\right\}$, we have to guarantee the detailed balance condition. The 
transition probability density $\mathrm{W}_{\mathrm{x}, \mathrm{y}}$ of going from state $\mathrm{x}$ to state $\mathrm{y}$ is,

$$
\mathrm{W}_{\mathrm{x}, \mathrm{y}}=\mathrm{W}_{\text {prop }}(\mathrm{x} \rightarrow \mathrm{y}) \mathrm{A}_{\mathrm{acc}}(\mathrm{x} \rightarrow \mathrm{y})
$$

Where, $\mathrm{W}_{\text {prop }}(\mathrm{x} \rightarrow \mathrm{y})$ is the proposal probability density and $\mathrm{A}_{\mathrm{acc}}(\mathrm{x} \rightarrow \mathrm{y})$ is the acceptance probability density of moving from $\mathrm{x}$ to $\mathrm{y}$ respectively.

Similarly, the transition probability $\mathrm{W}_{\mathrm{y}, \mathrm{x}}$ of going from state $\mathrm{y}$ to $\mathrm{x}$ is,

$$
\mathrm{W}_{\mathrm{y}, \mathrm{x}}=\mathrm{W}_{\text {prop }}(\mathrm{y} \rightarrow \mathrm{x}) \mathrm{A}_{\mathrm{acc}}(\mathrm{y} \rightarrow \mathrm{x})
$$

Furthermore, the proposal probability density $\mathrm{W}_{\text {prop }}(\mathrm{x} \rightarrow$ y) of inserting a time vertex $\left(\tau_{n+1}\right)$ is given by the probability of picking the imaginary time location $\tau_{n+1}$ :

$$
\mathrm{W}_{\text {prop }}(\mathrm{x} \rightarrow \mathrm{y})=\frac{\mathrm{d} \tau}{\beta}
$$

On the other hand, the proposal probability of removing a time vertex is just the one of selecting that particular vertex out of the $n+1$ available vertices:

$$
\mathrm{W}_{\text {prop }}(\mathrm{y} \rightarrow \mathrm{x})=\frac{1}{\mathrm{n}+1}
$$

Choosing the acceptance probabilities $\mathrm{A}_{\mathrm{acc}}(\mathrm{x} \rightarrow \mathrm{y})$ and $\mathrm{A}_{\mathrm{acc}}(\mathrm{y} \rightarrow \mathrm{x})$ such that

$$
\begin{aligned}
& \frac{\mathrm{W}_{\mathrm{x}, \mathrm{y}}}{\mathrm{W}_{\mathrm{y}, \mathrm{x}}}=\frac{\mathrm{d} \tau}{\beta} \frac{\mathrm{n}+1}{1} \frac{\mathrm{A}_{\mathrm{acc}}(\mathrm{x} \rightarrow \mathrm{y})}{\mathrm{A}_{\mathrm{acc}}(\mathrm{y} \rightarrow \mathrm{x})} \\
& =\frac{\mathrm{p}(\mathrm{y})}{\mathrm{p}(\mathrm{x})} \frac{\mathrm{A}_{\mathrm{acc}}(\mathrm{x} \rightarrow \mathrm{y})}{\mathrm{A}_{\mathrm{acc}}(\mathrm{y} \rightarrow \mathrm{x})}=\frac{\mathrm{p}(\mathrm{y})}{\mathrm{p}(\mathrm{x})} \frac{1 /(\mathrm{n}+1)}{\mathrm{d} \tau / \beta}
\end{aligned}
$$

Applying Metropolis' algorithm to fulfill detailed balance we obtain the acceptance ratio as,

$$
\begin{aligned}
\mathrm{A}_{\mathrm{acc}} & =\min \left[1, \frac{\mathrm{p}(\mathrm{y}) \mathrm{W}_{\operatorname{prop}}(\mathrm{y} \rightarrow \mathrm{x})}{\mathrm{p}(\mathrm{x}) \mathrm{W}_{\operatorname{prop}}(\mathrm{x} \rightarrow \mathrm{y})}\right] \\
& =\min \left[1, \frac{1}{\mathrm{n}+1} \frac{\beta}{\mathrm{d} \tau} \frac{\mathrm{p}(\mathrm{y})}{\mathrm{p}(\mathrm{x})}\right]
\end{aligned}
$$

The configuration probability densities ratio $\mathrm{p}(\mathrm{y}) / \mathrm{p}(\mathrm{x})$ is infinitesimally small, the transition rate from configuration $\mathrm{x}$ to $\mathrm{y}$ remains finite [55]. The partition function is computed through the CT-QMC hybridization algorithm for various configuration with weight $\omega(C)$. From which, we compute the Green's function of imaginary time or frequency.

\section{Hybridization-expansion CT-QMC:}

For this calculation, we have to focus on the simplest Anderson impurity model that can easily be generalized as the generic multi-orbital problems. To derive an expansion around the atomic limit, which could be understood as the expansion in the hybridization technique [56]. The effective action as the sum of an "atomic" term having all the local terms and a term with the hybridization to the bath as given by,

$$
\begin{gathered}
\mathrm{S}_{\mathrm{eff}}=\mathrm{S}_{\mathrm{loc}}+\sum_{\sigma} \int_{0}^{\beta} \mathrm{d} \tau \mathrm{d} \tau^{\prime} \mathrm{c}_{\sigma}^{\dagger}(\tau) \Delta_{\sigma}\left(\tau-\tau^{\prime}\right) \mathrm{d}_{\sigma}\left(\tau^{\prime}\right) \\
=\mathrm{S}_{\mathrm{loc}}+\sum_{\sigma} \mathrm{S}_{\mathrm{hyb}}^{\sigma}
\end{gathered}
$$

From Eq.(6), we get,

$$
\begin{gathered}
\mathrm{Z}_{=} \int \mathrm{D}\left[\mathrm{a}_{\sigma}^{\dagger}, \mathrm{a}_{\sigma}\right] \exp \left(-\mathrm{S}_{\mathrm{loc}}+\sum_{\sigma} \mathrm{S}_{\text {hyb }}^{\sigma}\right) \\
=\int \mathrm{D}\left[\mathrm{a}_{\sigma}^{\dagger}, \mathrm{a}_{\sigma}\right] \exp \left(-\mathrm{S}_{\mathrm{loc}}\right)\left[\operatorname{pod}_{\sigma} \frac{(-1)_{\sigma}^{\mathrm{n}}}{\mathrm{n}_{\sigma !}}\left(\mathrm{S}_{\text {hyb }}^{\sigma}\right)^{\mathrm{n}}\right]
\end{gathered}
$$

Using the idea of path integral QMC with stochastic series expansion [58], we may write,

$$
\begin{gathered}
\mathrm{Z}=\sum_{\mathrm{n} \uparrow, \mathrm{n} \downarrow=0}^{\infty}\left\langle\operatorname{Tr} \prod_{\sigma} \frac{(-1)_{\sigma}^{\mathrm{n}}}{\mathrm{n}_{\sigma !}}\left(\mathrm{S}_{\mathrm{hyb}}^{\sigma}\right)^{\mathrm{n}}\right\rangle_{\mathrm{loc}} \\
\mathrm{Z}=\sum_{\mathrm{n} \uparrow, \mathrm{n} \downarrow=0}^{\infty} \int_{0}^{\beta} \mathrm{d} \tau_{1}^{\sigma} \ldots \mathrm{d} \tau_{\mathrm{n} \uparrow}^{\prime \sigma} \int_{0}^{\beta} \mathrm{d} \tau_{1}^{\sigma^{\prime}} \ldots \mathrm{d} \tau_{\mathrm{n} \downarrow}^{\prime} \sigma^{\prime} \\
\prod_{\sigma} \frac{(-1)_{\sigma}^{\mathrm{n}}}{\mathrm{n}_{\sigma !}} \prod_{\mathrm{i}=1}^{\mathrm{n}_{\sigma}} \Delta_{\sigma}\left(\tau_{\mathrm{i}}^{\sigma}-\tau_{\mathrm{i}}^{\prime} \sigma\right) \\
\times \operatorname{Tr}\left[\mathrm{e}^{-\beta \mathscr{H} \mathscr{H}_{1} \mathrm{oc}} \operatorname{Tr} \prod_{\mathrm{i}=1}^{\mathrm{n} \uparrow} \mathrm{c}_{\uparrow}^{\dagger}\left(\tau_{\mathrm{i}}^{\sigma}\right) \mathrm{c}_{\uparrow}\left(\tau_{\mathrm{i}}^{\prime}{ }^{\prime}\right) \prod_{\mathrm{i}=1}^{\mathrm{n} \sigma^{\prime}} \mathrm{c}_{\downarrow}^{\dagger}\left(\tau_{\mathrm{i}}^{\sigma^{\prime}}\right) \mathrm{c}_{\downarrow}^{\dagger}\left(\tau_{\mathrm{i}}^{\prime} \sigma^{\prime}\right)\right]
\end{gathered}
$$

Which is the sum over many continuous variables and the product of hybridization functions times the trace involving spin up and spins down operators.

$$
\therefore \mathrm{Z}=\int_{\mathrm{C}} \omega(\mathrm{C}) \approx \Sigma_{\mathrm{C}}^{\mathrm{MC}} \operatorname{sign}(\omega \mathrm{C})
$$

Where $\omega(C)$ is the weights of configurations $C$ in the limit $\mathrm{N} \rightarrow \infty$ gives the probability,

$$
\begin{gathered}
\mathrm{p}(\mathrm{C})=\frac{1}{\mathrm{Z}} \omega(\mathrm{C}) \\
\therefore \mathrm{Z}=\frac{1}{\mathrm{Z}} \int_{\mathrm{C}} \omega(\mathrm{C}) \mathrm{f}(\mathrm{C}) \sim \frac{1}{\mathrm{Z}} \sum_{\mathrm{C}}^{\mathrm{MC}} \mathrm{f}(\mathrm{C}) \operatorname{sign}(\omega(\mathrm{C}))
\end{gathered}
$$

The weight of the configuration is computed as, 


$$
\begin{aligned}
\omega(\mathrm{C})= & \operatorname{Tr} \times \mathrm{e}^{-\beta \mathscr{G}_{\mathrm{i}} \mathrm{oc}} \operatorname{Tr} \prod_{\mathrm{i}=1}^{\mathrm{n} \uparrow} \mathrm{a}_{\uparrow}^{\dagger}\left(\tau_{\mathrm{i}}^{\sigma}\right) \mathrm{a}_{\uparrow}\left(\tau_{\mathrm{i}}^{\prime} \sigma\right) \\
& \prod_{i=1}^{n \downarrow} a_{\downarrow}^{\dagger}\left(\tau_{i}^{\sigma^{\prime}}\right) a_{\downarrow}\left(\tau_{i}^{\prime} \sigma^{\prime}\right) \\
\times & \prod_{\sigma} \frac{(-1)_{\sigma}^{\mathrm{n}}}{\mathrm{n}_{\sigma !}} \prod_{\mathrm{i}=1}^{\mathrm{n}_{\sigma}} \Delta_{\sigma}\left(\tau_{\mathrm{i}}^{\sigma}-\tau_{\mathrm{i}}^{\sigma}\right)
\end{aligned}
$$

Unfortunately, these calculations have alternating signs problems (-ve sign problem) For evaluating the partition function, we start from a imaginary time step diagram and sum of the possible permutations of the fluctuations.

\section{Computing Green's Function from Partition Function:}

The impurity Green's function could also be regarded as 8the logarithmic derivative of $\mathrm{Z} w$. r. to the hybridization, $\Delta(\tau)$ as given by,

$$
\begin{gathered}
\mathrm{G}_{\sigma}(\tau)=-\frac{1}{\beta} \frac{\delta \log \mathrm{Z}}{\delta \Delta_{\sigma}(-\tau)}\left[\because \mathrm{G}(\tau)=-\frac{\delta \log \mathrm{Z}}{\delta \Delta(\tau)}\right] \\
\mathrm{G}_{\uparrow}(\tau)=-\frac{1}{\mathrm{Z} \beta} \int_{\mathrm{C}} \frac{\delta \operatorname{det} \Delta_{\uparrow \mathrm{C}}}{\delta \Delta_{\uparrow}(-\tau)} \times(-1)^{\mathrm{n} \uparrow+\mathrm{n} \downarrow} \operatorname{det} \Delta_{\downarrow \mathrm{C}} \operatorname{Tr} \mathrm{C}
\end{gathered}
$$

Each configuration give contributions for a discrete set of imaginary times:

$$
\begin{gathered}
\mathrm{G}_{\sigma}(\tau) \sim-\frac{1}{\mathrm{Z} \beta} \sum_{\mathrm{C}}^{\mathrm{MC}} \sum_{\mathrm{k}, \mathrm{l}} \delta\left(\tau_{\mathrm{k}}^{\sigma}-\tau_{\mathrm{l}}^{\prime}{ }^{\prime}+\tau\right) \times\left[\Delta_{\sigma \mathrm{C}}^{-1}\right]_{\mathrm{k}, 1} \\
\times \operatorname{sign}(\omega(\mathrm{C}))
\end{gathered}
$$

In fact, these contributions can be computed by considering a very fine imaginary time slices. This creates a high frequency noise in Matsubara frequencies. To compute single particle Green's function in Legendre representation, we consider the single-particle imaginary time Green's function $\mathrm{G}(\tau)$ defined on the interval $[0, \beta][57$, 58]. Expanding $\mathrm{G}(\tau)$ in terms of the Legendre polynomials $\mathrm{P}_{1}(\mathrm{x})$, Fig. 3 (left) which are considered to be the basis for expressing the function defined over an interval $[-1,1]$ to calculate the imaginary-time Green's function as,

$$
\mathrm{G}(\tau)=\sum_{\mathrm{l} \geq 0} \frac{\sqrt{2 \mathrm{l}+1}}{\beta} \mathrm{P}_{\mathrm{r}}[\mathrm{x}(\tau)] \mathrm{G}_{\mathrm{l}}
$$

Where, $\mathrm{x}(\tau)=\frac{2 \tau}{\beta}-1$ and $G_{l}$ is the Legendre coefficients that decays very quickly, which may be defined as,

$$
\mathrm{G}_{\mathrm{l}}=\sqrt{2 \mathrm{l}+1} \int_{0}^{\beta} \mathrm{d} \tau \mathrm{G}(\tau) \mathrm{P}_{\mathrm{l}}[\mathrm{x}(\tau)]
$$

The number of Legendre coefficients considered to be important for the accurate representation of a given observable under consideration and is difficult to infer from looking at the coefficients themselves. Recently, it has been proposed to improve this method by employing the Kernel polynomial method with model independent basis, what is termed as intermediate representation (IR) basis [59]. Truncating the Legendre coefficients that are zero within their error bars can reduce the Matsubara frequency noise. The imaginary part of Green's function of Matsubara frequency vs. Matsubara frequency is shown in Fig.3 (right), which is one of the typical outcomes of this method of calculation.

\section{Green's Functions, Self-Energy and Spectral Function:}

The dynamical equation of motion (Dyson equation):

$$
\begin{gathered}
\mathrm{G}_{\sigma}^{-1}\left(\mathrm{i} \omega_{v}\right)=\mathscr{G}_{0}^{-1}\left(\mathrm{i} \omega_{v}\right)-\Sigma_{\sigma}\left(\mathrm{i} \omega_{v}\right) \\
=\mathrm{i} \omega_{v}+\mu-\varepsilon_{\mathbf{k}}-\Sigma_{\sigma}\left(\mathrm{i} \omega_{v}\right)
\end{gathered}
$$

Where, $\Sigma_{\sigma}\left(\mathrm{i} \omega_{v}\right)$ self-energy of Matsubara frequency and $\omega_{v}=\left[\frac{(2 v+1) \pi}{\beta}\right]$ is the Matsubara frequency [60]. In the DMFT limit, the self-energy $\Sigma_{\sigma}$ becomes a local quantity and the lattice Green's function retains its momentum-dependence via the non-interacting lattice dispersion $\varepsilon_{k}$.

The momentum dependent lattice Green's function in the DMFT limit is,

$$
\mathrm{G}_{\sigma}\left(\mathrm{k}, \mathrm{i} \omega_{v}\right)=\frac{1}{\mathrm{i} \omega_{v}+\mu-\varepsilon_{\mathbf{k}}-\Sigma_{\sigma}\left(\mathrm{i} \omega_{v}\right)}
$$

Which leads to the equation for the effective Weiss field,

$$
\begin{aligned}
& \mathscr{G}_{0}^{-1}\left(\mathrm{i} \omega_{v}\right)=\Sigma_{\sigma}\left(\mathrm{i} \omega_{v}\right)+\mathrm{G}_{\sigma}^{-1}\left(\mathrm{i} \omega_{v}\right) \\
= & \Sigma_{\sigma}\left(\mathrm{i} \omega_{v}\right)+\left[\int \frac{\mathrm{D}(\varepsilon)}{\mathrm{i} \omega_{v}+\mu-\varepsilon_{\mathbf{k}}-\Sigma_{\sigma}\left(\mathrm{i} \omega_{v}\right)}\right]^{-1}
\end{aligned}
$$

Where, $\mathrm{D}(\varepsilon)$ is the non-interacting density of states of the original lattice. And the imaginary time (thermal) Green function,

$$
\mathrm{G}_{\mathrm{ij}, \sigma}(\tau)=-\left\langle\mathrm{Tc}_{\mathrm{i} \sigma}(\tau) \mathrm{c}_{\mathrm{j} \sigma^{\prime}}^{\dagger}(0)\right\rangle \rightarrow \Sigma_{\sigma}\left(\mathrm{i} \omega_{v}\right)
$$

Where $\mathrm{T}$ is the time-ordering operator w.r.to t or $\tau$ and $\mathrm{c}_{\mathrm{i} \sigma}=\exp (\mathrm{iH} \tau) \mathrm{c}_{\mathrm{il}} \exp (-\mathrm{iH} \tau)$ in both cases [61, 62]. The Fourier transform $\mathrm{G}\left(\mathrm{i} \omega_{v}\right)$ of $\mathrm{G}_{\mathrm{ij}, \sigma}(\tau)$ and the Matsubara self-energy are computed as,

$$
\Sigma_{\sigma}\left(\mathrm{i} \omega_{v}\right)=\mathscr{G}_{0}^{-1}\left(\mathrm{i} \omega_{v}\right)-\mathrm{G}_{\sigma}^{-1}\left(\mathrm{i} \omega_{v}\right)
$$

This is the most expensive part of the calculation, which actually provides the new Green's function, $\mathscr{G}_{0}$ 

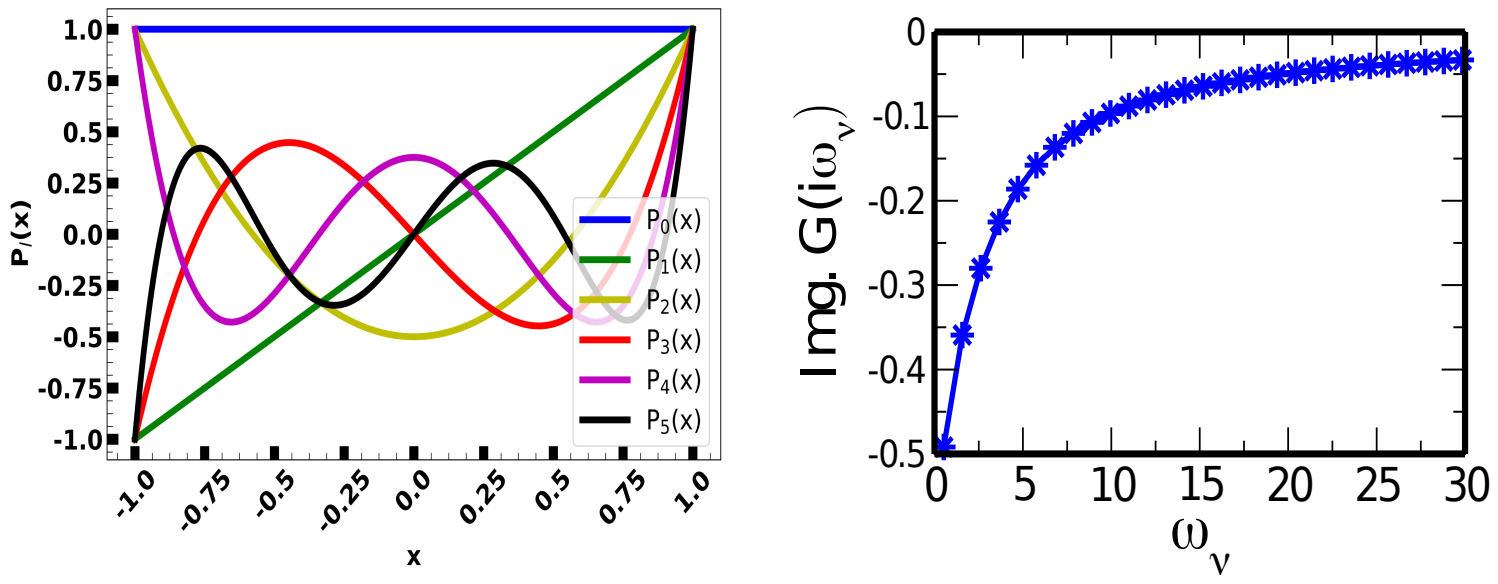

FIGURE 3. (Color online) First five orders of Legendre's polynomials of x (left). A typical outcome of calculation of Green's function of Matsubara frequency (right).

for the next iteration of self-consistency calculation [63, 64]. In the non-interacting system, the spectral function at a given momentum is a Dirac $\delta$-function at $\omega=\varepsilon_{\mathrm{k}}$,

$$
\mathrm{A}(\mathrm{k}, \omega)=\delta\left(\omega-\varepsilon_{\mathrm{k}}\right)
$$

The possible excitation of the system is encoded in the interacting Green's function on the real axis as given by,

$$
\mathrm{G}^{\mathrm{R}}(\mathrm{k}, \omega)=\frac{1}{\omega+\mu-\varepsilon(\mathrm{k})+\Sigma^{\mathrm{R}}(\mathrm{k}, \omega)}
$$

with the corresponding spectral function is,

$$
\mathrm{A}(\mathrm{k}, \omega)=-\frac{1}{\pi} \operatorname{Im}\left[\mathrm{G}^{\mathrm{R}}(\mathrm{k}, \omega)\right]
$$

In the infinite coordination limit, the momentum dependence of the self-energy $\Sigma(k, \omega) \simeq \Sigma(\omega)$.The solution of a quantum many body systems may be obtained as the solution of a quantum impurity model subject to an appropriately defined self-consistency condition. Thus, the spectral function simply be expressed as,

$$
\mathrm{A}(\omega)=-\frac{1}{\pi} \operatorname{Im}\left[\mathrm{G}^{\mathrm{R}}(\omega)\right]
$$

The usual bandstructure (spaghetti plot) is replaced by a plot of the above spectral function, which can be compared with the experimental results of angular-resolved photoemission spectroscopy (ARPES) [13, 65]. Turning on correlations, the spectral function has a Lorentzian profile as,

$$
\begin{gathered}
\mathrm{A}(\mathrm{k}, \omega)= \\
-\frac{1}{\pi} \frac{\operatorname{Im} \Sigma^{\mathrm{R}}(\mathrm{k}, \omega)}{\left[\omega+\mu-\varepsilon(\mathrm{k})+\operatorname{Re} \Sigma^{\mathrm{R}}(\mathrm{k}, \omega)\right]^{2}+\left[\Sigma^{\mathrm{R}}(\mathrm{k}, \omega)\right]^{2}}
\end{gathered}
$$

The imaginary part of the self-energy introduces a broadening of the original Delta-function like excitation, whereas the real part is responsible for a shift of excitation energies. The program can treat data that can be expressed as the periodic imaginary time Green's function as,

$$
\mathrm{G}\left(\mathrm{i} \omega_{v}\right)=\int_{-\infty}^{\infty} \mathrm{e}^{\mathrm{i} \omega_{v}} \mathrm{G}(\tau) \mathrm{d} \tau=\int_{-\infty}^{\infty} \frac{\mathrm{d} \omega}{2 \pi}\left(\frac{\mathrm{A}(\omega)}{\mathrm{i} \omega_{\mathrm{n}}-\omega}\right)
$$

Or,

$$
\mathrm{G}(\tau)=\int_{-\infty}^{\infty} \frac{\mathrm{d} \omega}{2 \pi}\left(\frac{\mathrm{e}^{-\omega \tau} \mathrm{A}(\omega)}{1 \pm \mathrm{e}^{-\beta \omega}}\right),
$$

Where $\omega_{v}$ is the Matsubara frequency. The Green's function $\mathrm{G}(\tau)$ or $G(\omega)$ as QMC data, we further apply analytic continuation method to obtain the real-frequency data, $A(\omega)$ using Maximum entropy model. The Maximum entropy model algorithm $[66,67,68]$ is based on the Bayes theorem as given by,

$$
\mathrm{P}(\mathrm{A} \mid \mathrm{G})=\frac{\mathrm{P}(\mathrm{G} \mid \mathrm{A}) \mathrm{P}(\mathrm{A})}{\mathrm{P}(\mathrm{G})} \propto \mathrm{e}^{\left(-\frac{\chi^{2}}{2}-\alpha \mathrm{S}\right)},
$$

Where,

$$
\chi^{2}=\sum_{\mathrm{i}} \frac{\left(\mathrm{G}_{\mathrm{i}}-\overline{\mathrm{G}}_{\mathrm{i}}\right)^{2}}{\sigma_{\mathrm{i}}^{2}} .
$$

with $\bar{G}=\mathbf{K} A$ And the differential entropy of information is,

$$
\mathrm{S}=-\int \frac{\mathrm{d} \omega}{2 \pi} \mathrm{A}(\omega) \ln \frac{\mathrm{A}(\omega)}{\mathrm{D}(\omega)}
$$

Where $D(\omega)$, is called the default model. For maximizing the posterior probability, we need to choose the optimal 
value of $\alpha$ using the Bayesian inference $[24,68]$ as,

$$
\mathrm{P}(\alpha \mid \mathrm{G}) \propto \frac{\mathrm{P}(\alpha)}{\mathrm{Z}_{\alpha}^{\mathrm{S}}} \int \mathscr{D} \mathrm{A} \mathrm{e}^{\alpha \mathrm{S}-\frac{\chi^{2}}{2}},
$$

The value of $\alpha$ is then taken as the most probable one. To find the maximum, one needs a guess for the prior $P(\alpha)$.

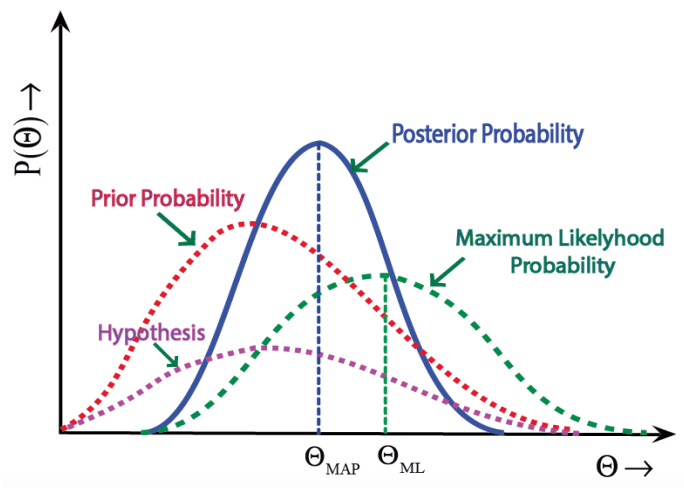

FIGURE 4. (Color online) The maximum a posteriory $\Theta_{M A P}$ is obtained through the Bayesian statistical inferences [24]

The maximum posterior probability is regarded as the realistic representation of data for the real materials. It is estimated through Bayesian theorem out of the given conditional probabilities by maximizing the entropy of information as shown in Fig.4. We have used density functional theory to calculate the density of states (DOS), which is the input data for DMFT calculation. DMFT generates the Green's function data in imaginary time or Matsubara frequency through CT-QMC algorithm with hybridization expansion, which is considered to be a rough data. The real frequency data (spectral density) are computed from the Green's function data using Maximum Entropy model, which is equivalent to the experimental observation. By analyzing spectral density, MIT is predicted with the adjustment parameters $\mathrm{U}, \beta$ and $\mathrm{J}$ values.

\section{RESULTS AND DISCUSSION}

The electronic structure of $\mathrm{La}_{\mathrm{x}} \mathrm{Sr}_{1-\mathrm{x}} \mathrm{VO}_{3}(0<\mathrm{x}<1)$ system has been studied through the conventional DFT along with the DMFT. This system is the typical examples of the extended transition metal oxide system with a $3 d^{1}$ electronic configuration. The supercell of $\mathrm{SrVO}_{3}$ is reconstructed within the frameworks of $1 \times 1 \times 2$ for the abinitio calculation. With the k-mesh grid, the Monk-horst pack of $11 \times 11 \times 5$, the convergence criteria are chosen to be $10^{-3} \mathrm{e}, 10^{-5} \mathrm{eV}$ and $0.05 \mathrm{eV} /{ }^{0} A$ respectively $[69,70$, 71]. The optimized lattice parameter $4.12{ }^{\circ} A$ and crystal structure for the sample is in Fig. 5. The conventional
DFT results for DOS and Band structure of $\mathrm{La}_{\mathrm{x}} \mathrm{Sr}_{1-\mathrm{x}} \mathrm{VO}_{3}$ show the sample is metallic as in Fig.6, which is in agreement with others works [72]. The outcomes of the numerical simulation are discussed here as the followings:

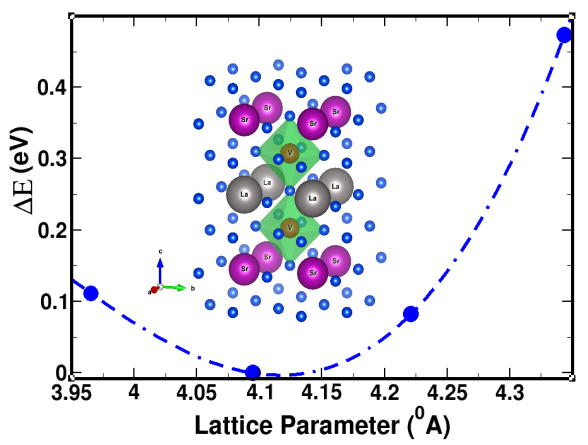

FIGURE 5. (Color online)(a) Lattice parameter optimization curve with crystal structure of $\mathrm{La}_{\mathrm{x}} \mathrm{Sr}_{1-\mathrm{x}} \mathrm{VO}_{3}$

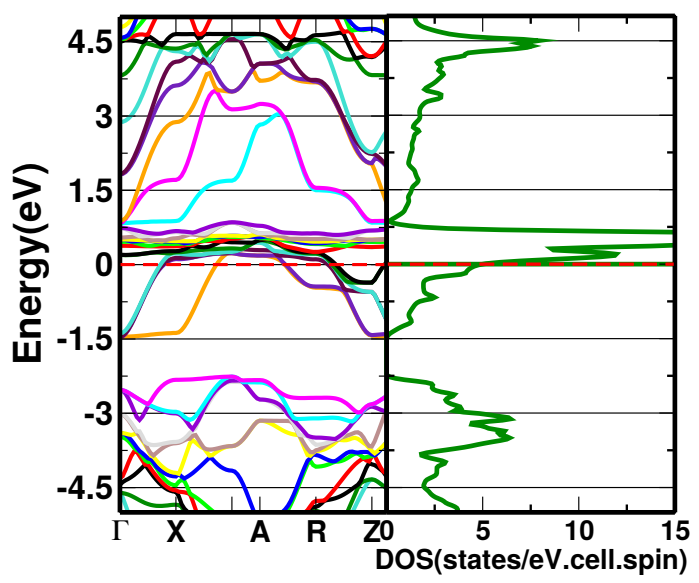

FIGURE 6. (Color online) The electronic structure (DOS and Bandstructure) of $\mathrm{La}_{\mathrm{x}} \mathrm{Sr}_{1-\mathrm{x}} \mathrm{VO}_{3}$

The characteristic QMC data, such as $\mathrm{G}(\tau)$ or $\mathrm{G}(\omega)$ are obtained through CT-QMC with hybridization techniques as shown in Fig. 7-10 for $\mathrm{La}_{\mathrm{x}} \mathrm{Sr}_{1-\mathrm{x}} \mathrm{VO}_{3}$ system. These data are used for analyzing Mott-Hubbard metal insulator transition at first hand. In general, the QMC data are noisy, incomplete and over-sampled one, so we need to use analytic continuation (reconstruction of data) to obtain the real frequency data for comparing the experimental results. We have used Maximum Entropy model based upon the Bayesian statistical inferences for the analytic continuation [73, 74]. In fact, it gives the spectral function $\mathrm{A}(\omega)$ along with the error estimation of calculation.

The real frequency spectral function $\mathrm{A}(\omega)$ is computed using the QMC data through Maximum entropy model (MEM), which works on the Bayesian statistical 


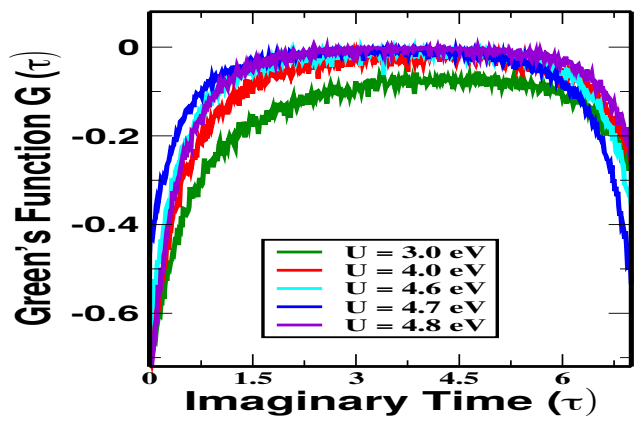

FIGURE 7. (Color online) The Green's Function vs. imaginary time $(\tau)$ of $\mathrm{La}_{0.67} \mathrm{Sr}_{0.33} \mathrm{VO}_{3}$ with various $\mathrm{U}$ values

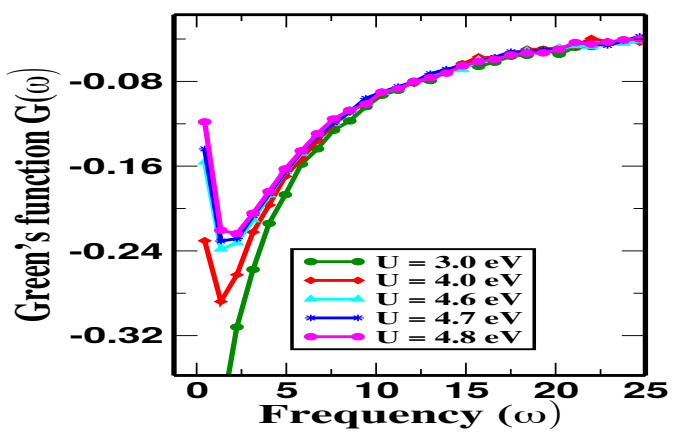

FIGURE 8. (Color online) Green's Function vs. frequency $(\omega)$ of $\mathrm{La}_{0.67} \mathrm{Sr}_{0.33} \mathrm{VO}_{3}$ is the Fourier transform of $\mathrm{G}(\tau)$

inferences $[75,76]$. The metal insulator transition for

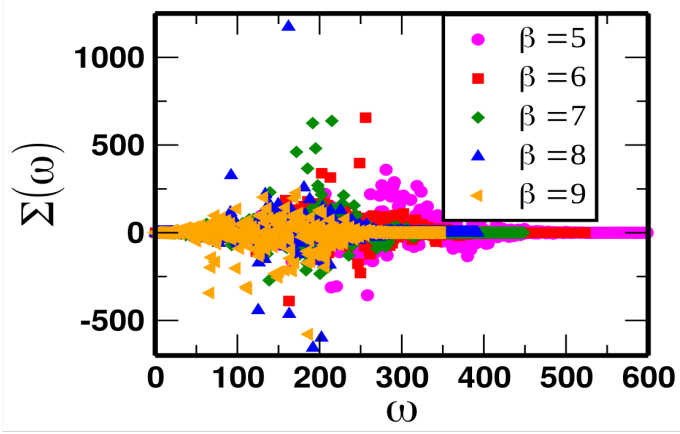

FIGURE 9. (Color online) The self-energy vs. frequency $(\omega)$ for $\mathrm{U}=3.5 \mathrm{eV}$ and various $\beta$ values

$\mathrm{La}_{0.33} \mathrm{Sr}_{0.67} \mathrm{VO}_{3}$ system is computed for $\mathrm{U}=4.5 \mathrm{eV}$, and as in Fig. 11 which may vary with other parameters such as Hund's coupling (J) and bandwidth (W) as well.

The optimal value of hyperparameter or adjustable parameter $(\alpha)$ is crucial to represent the real data of the materials. For choosing the optimal value of $\alpha$ we use the logistic regression method for the reconstruction of data

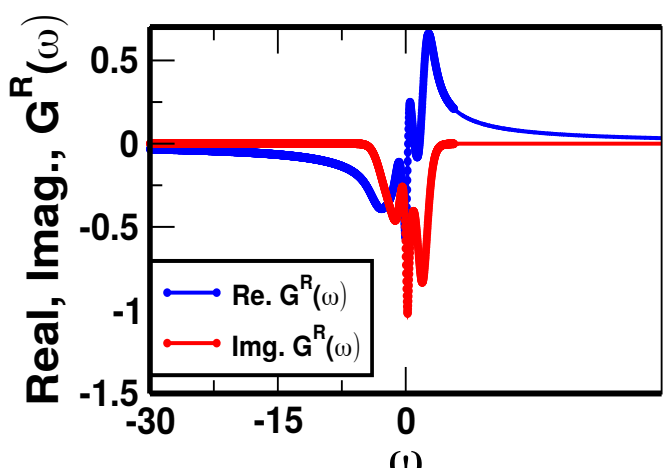

FIGURE 10. (Color online) The spectral function vs. frequency $(\omega)$ for $\mathrm{U}=3.5 \mathrm{eV}$ and various $\beta$ values of $\mathrm{La}_{0.33} \mathrm{Sr}_{0.67} \mathrm{VO}_{3}$ system.

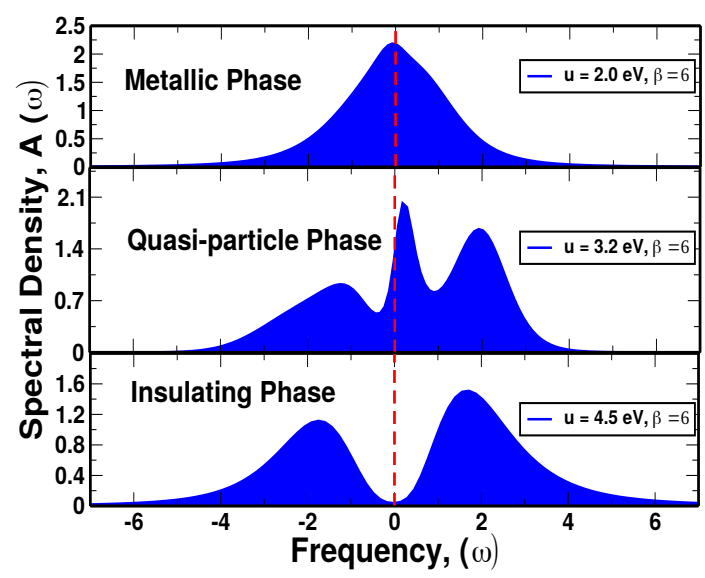

FIGURE 11. (Color online)The variation of spectral function $\mathrm{A}(\omega)$ with $\mathrm{U}$ values for the optimal value of $\alpha$

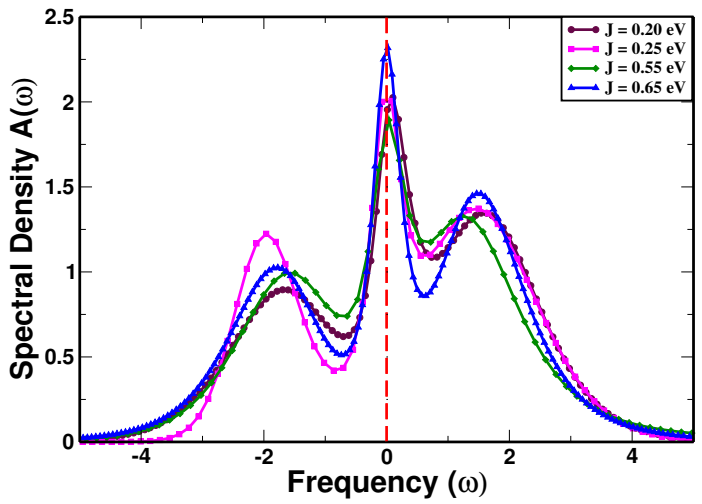

FIGURE 12. (Color online) The distribution of spectral function $\mathrm{A}(\omega)$ with the various values of $\mathrm{J}$.

$A(\omega)$ from the QMC data $G(\tau)$. The lower part of the information-fitting region as in Fig. 13 are chosen to be 


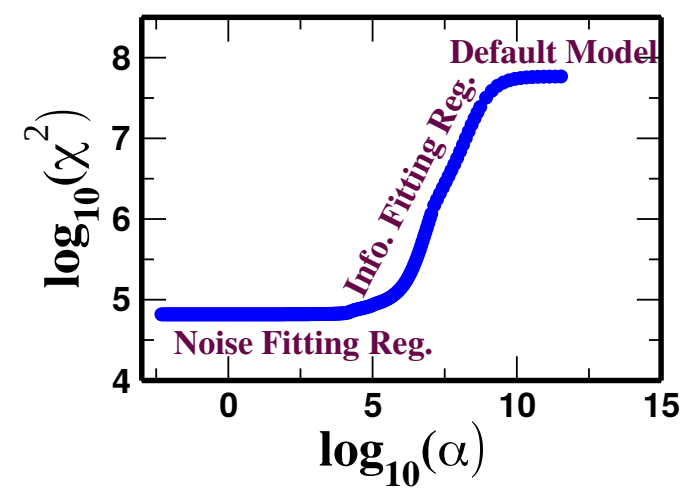

FIGURE 13. (Color online) The logistic regression curve for choosing the optimal value of for better representation of data obtained through MEM

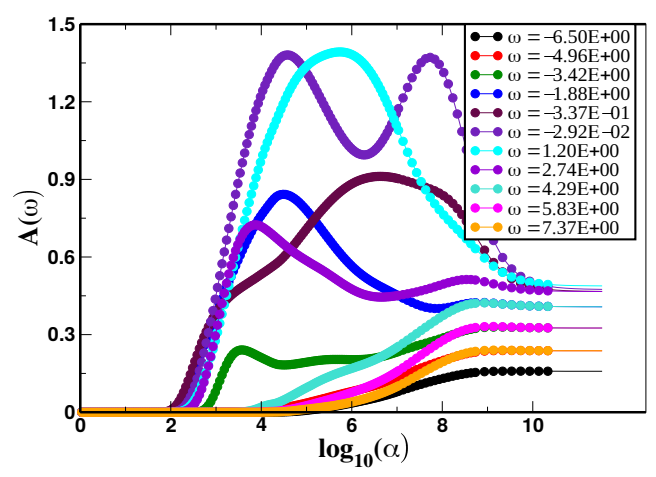

FIGURE 14. (Color online) The distribution of spectral function $\mathrm{A}(\omega)$ with the sample frequencies for the optimal value of $\alpha$.

optimal value of $\alpha$, which is determined by the maximum value of the curvature of $\log _{10} \chi^{2}$ vs. $\gamma \log _{10} \alpha$ curve [77]. We may also use sample frequencies for predicting the optimal values of $\alpha$ as in Fig. 14 . The statistical error

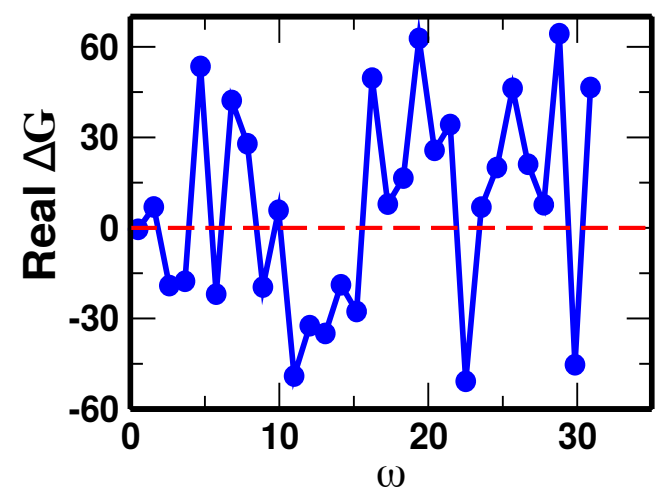

FIGURE 15. (Color online) The normalized deviation (ND) of real part of $\Delta G$ for the data computed with MEM

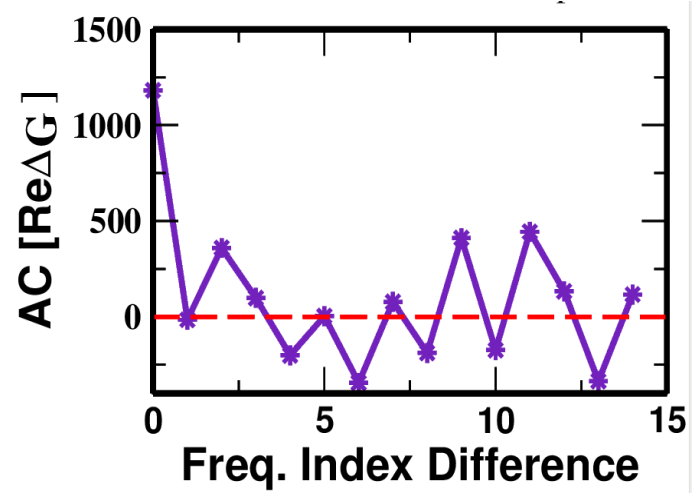

FIGURE 16. (Color online) The autocorrelation (AC) of real part of $\Delta G$ for the data computed with MEM.

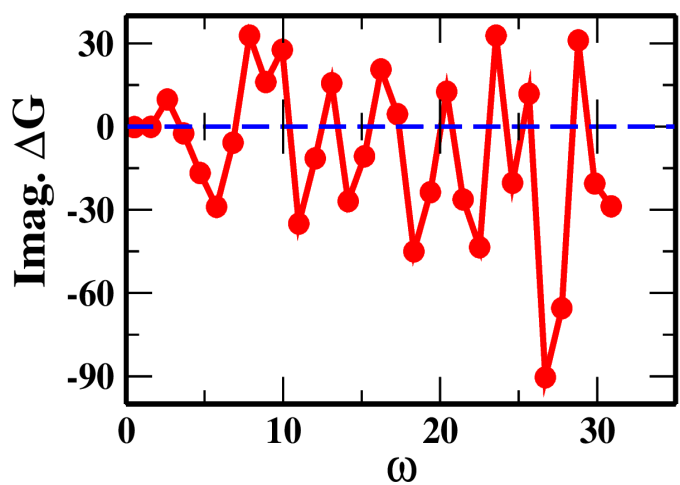

FIGURE 17. (Color online) The normalized deviation (ND) of imaginary part of $\Delta G$ for the data computed with MEM.

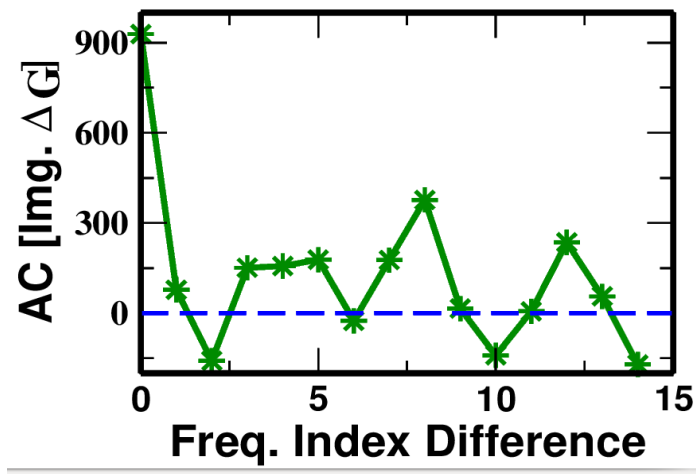

FIGURE 18. (Color online) The autocorrelation (AC) of imaginary part of $\Delta G$ for the data computed with MEM.

such as the normalized deviation of real and imaginary parts of the uncertainty of Green's function of frequency $\Delta G$ and its auto correlation are computed within the permissible standard error bar Fig. 15-18 [78, 79, 80, 81, 82, 83]. 


\section{CONCLUSIONS}

We review the theoretical concept of DFT +DMFT approximation to calculate the electronic properties of strongly correlated system through CT-QMC algorithm as an impurity solver. Implementation of Maximum Entropy model for the real frequency data (spectral function) to investigate the Mott-Hubbard MIT is also discussed. From the calculation, the lattice parameters of optimized $\mathrm{La}_{\mathrm{x}} \mathrm{Sr}_{1-\mathrm{x}} \mathrm{VO}_{3}$ are $\mathrm{a}=4.12{ }^{0} \mathrm{~A}, \mathrm{~b}=4.12{ }^{0} \mathrm{~A}$ and $\mathrm{c}=$ $7.93^{0} \mathrm{~A}$. The electronic band structure from conventional DFT shows metallic behavior of the sample. However, after the application of DMFT with CT-QMC hybridization expansion it turns out to be a Mott insulator at $\mathrm{U}=$ $4.5 \mathrm{eV}$, and $\beta=6(\mathrm{eV})^{-1}$. The optimal value of adjustable parameter $\alpha$ is obtained through logistic regression. Statistical error confirms that present calculation are within the permissible error bar limit.

\section{ACKNOWLEDGMENTS}

The authors are gratefully acknowledged to UGC and NAST for their financial supports. CDP of TU and HPC of $\mathrm{KU}$ are also acknowledged for their computational resources. We also acknowledge the ALPS open source library for our calculation.

\section{EDITOR'S NOTE}

This manuscript was submitted to the Association of Nepali Physicists in America (ANPA) Conference 2021 for publication in the special issue of Journal of Nepal Physical Society.

\section{REFERENCES}

1. K Held. Electronic structure calculations using dynamical mean field theory. Advances in physics, 56(6):829-926, 2007.

2. Walter Metzner and Dieter Vollhardt. Correlated lattice fermions in d $=\infty$ dimensions. Physical review letters, 62(3):324, 1989

3. Nevill F Mott. The basis of the electron theory of metals, with special reference to the transition metals. Proceedings of the Physical Society. Section A, 62(7):416, 1949.

4. Masatoshi Imada, Atsushi Fujimori, and Yoshinori Tokura. Metalinsulator transitions. Reviews of modern physics, 70(4):1039, 1998

5. C Bell, S Harashima, Y Kozuka, M Kim, Bog G Kim, Y Hikita, and HY Hwang. Dominant mobility modulation by the electric field effect at the laalo 3/srtio 3 interface. Physical review letters, 103(22):226802, 2009.

6. Minjae Kim and BI Min. Nature of itinerant ferromagnetism of srruo 3: A dft+ dmft study. Physical Review B, 91(20):205116, 2015 .
7. S Streltsov, II Mazin, and K Foyevtsova. Localized itinerant electrons and unique magnetic properties of srru 2 o 6 . Physical Review $B, 92(13): 134408,2015$.

8. Alexey N Rubtsov, Vladimir V Savkin, and Alexander I Lichtenstein. Continuous-time quantum monte carlo method for fermions. Physical Review B, 72(3):035122, 2005.

9. Andreas Dirks, Philipp Werner, Mark Jarrell, and Thomas Pruschke. Continuous-time quantum monte carlo and maximum entropy approach to an imaginary-time formulation of strongly correlated steady-state transport. Physical Review E, 82(2):026701, 2010 .

10. B Chatterjee, J Skolimowski, K Makuch, and K Byczuk. Real-space dynamical mean-field theory of friedel oscillations in strongly correlated electron systems. Physical Review B, 100(11):115118, 2019.

11. Nicolaus Parragh, Alessandro Toschi, Karsten Held, and Giorgio Sangiovanni. Conserved quantities of s u (2)-invariant interactions for correlated fermions and the advantages for quantum monte carlo simulations. Physical Review B, 86(15):155158, 2012.

12. Alexander Kowalski, Andreas Hausoel, Markus Wallerberger, Patrik Gunacker, and Giorgio Sangiovanni. State and superstate sampling in hybridization-expansion continuous-time quantum monte carlo. Physical Review B, 99(15):155112, 2019.

13. Akira Sekiyama and Shigemasa Suga. High-energy bulk-sensitive angle-resolved photoemission study of strongly correlated systems. Journal of electron spectroscopy and related phenomena, 137:681685, 2004.

14. JH Shim, Kristjan Haule, and G Kotliar. X-ray absorption branching ratio in actinides: Lda+ dmft approach. EPL (Europhysics Letters), 85(1): 17007, 2009.

15. Gabriel Kotliar and Dieter Vollhardt. Strongly correlated materials: Insights from dynamical mean-field theory. Physics today, 57(3):53-60, 2004.

16. VI Anisimov, DE Kondakov, AV Kozhevnikov, IA Nekrasov, ZV Pchelkina, JW Allen, S-K Mo, H-D Kim, P Metcalf, S Suga, et al. Full orbital calculation scheme for materials with strongly correlated electrons. Physical Review B, 71(12):125119, 2005.

17. Frank Lechermann, Alexander I Lichtenstein, and Michael Potthoff. Realistic many-body approaches to materials with strong nonlocal correlations. The European Physical Journal Special Topics, 226(11):2591-2613, 2017.

18. B Amadon, F Lechermann, A Georges, F Jollet, TO Wehling, and AI Lichtenstein. Plane-wave based electronic structure calculations for correlated materials using dynamical mean-field theory and projected local orbitals. Physical Review B, 77(20):205112, 2008.

19. F Aryasetiawan, M Imada, A Georges, G Kotliar, S Biermann, and AI Lichtenstein. Frequency-dependent local interactions and low-energy effective models from electronic structure calculations. Physical Review B, 70(19):195104, 2004.

20. Antoine Georges, Gabriel Kotliar, Werner Krauth, and Marcelo J Rozenberg. Dynamical mean-field theory of strongly correlated fermion systems and the limit of infinite dimensions. Reviews of Modern Physics, 68(1):13, 1996.

21. Sandro Sorella. Generalized lanczos algorithm for variational quantum monte carlo. Physical Review B, 64(2):024512, 2001.

22. Daisuke Tahara and Masatoshi Imada. Variational monte carlo method combined with quantum-number projection and multivariable optimization. Journal of the Physical Society of Japan, 77(11):114701, 2008

23. Jan M Tomczak and Silke Biermann. 8 scientific highlight of the month:" optical properties of correlated materials-or why intelligent windows may look dirty".

24. Dominic Bergeron and A-MS Tremblay. Algorithms for optimized maximum entropy and diagnostic tools for analytic continuation. Physical Review E, 94(2):023303, 2016.

25. Dominic Bergeron. mmaxent user guide. 2016. 
26. Pavlo Zubko, Stefano Gariglio, Marc Gabay, Philippe Ghosez, and Jean-Marc Triscone. Interface physics in complex oxide heterostructures. Annu. Rev. Condens. Matter Phys., 2(1):141-165, 2011.

27. Motoaki Hirayama, Takashi Miyake, and Masatoshi Imada. Ab initio low-energy model of transition-metal-oxide heterostructure laalo3/srtio3. Journal of the Physical Society of Japan, 81(8):084708, 2012.

28. AV Mahajan, DC Johnston, DR Torgeson, and F Borsa. Magnetic and electronic properties of laxsr1-xvo3. Physica C: Superconductivity, 185:1195-1196, 1991.

29. Igor A Nekrasov, Georg Keller, DE Kondakov, AV Kozhevnikov, Th Pruschke, Karsten Held, Dieter Vollhardt, and VI Anisimov. Comparative study of correlation effects in ca v o 3 and sr v o 3 . Physical Review B, 72(15):155106, 2005.

30. Marcus Kollar. The foundations of dynamical mean-field theory. 2018.

31. Ciro Taranto, M Kaltak, N Parragh, G Sangiovanni, Georg Kresse, A Toschi, and K Held. Comparing quasiparticle g w+ dmft and lda+ dmft for the test bed material srvo 3. Physical Review B, 88(16):165119, 2013.

32. Peter Anders, Emanuel Gull, Lode Pollet, Matthias Troyer, Philipp Werner, Michael Bachmann, and Ofer Biham. Diagrammatic monte carlo method for bosonic impurity problems. Chem. Phys, 127:144703, 2007.

33. JE Gubernatis, Mark Jarrell, RN Silver, and DS Sivia. Quantum monte carlo simulations and maximum entropy: Dynamics from imaginary-time data. Physical Review B, 44(12):6011, 1991.

34. Matthew D Pickett, Gilberto Medeiros-Ribeiro, and R Stanley Williams. A scalable neuristor built with mott memristors. Nature materials, 12(2):114-117, 2013.

35. Karsten Held, IA Nekrasov, G Keller, Volker Eyert, Nils Blümer, AK McMahan, RT Scalettar, Th Pruschke, VI Anisimov, and Dieter Vollhardt. Realistic investigations of correlated electron systems with lda+ dmft. physica status solidi (b), 243(11):2599-2631, 2006.

36. Antoine Georges, Gabriel Kotliar, Werner Krauth, and Marcelo J Rozenberg. Dynamical mean-field theory of strongly correlated fermion systems and the limit of infinite dimensions. Reviews of Modern Physics, 68(1):13, 1996.

37. John Hubbard. Electron correlations in narrow energy bands. Proceedings of the Royal Society of London. Series A. Mathematical and Physical Sciences, 276(1365):238-257, 1963.

38. Steffen Backes. Density functional theory and dynamical meanfield theory. a way to model strongly correlated systems. 2017.

39. Emilia Morosan, Douglas Natelson, Andriy H Nevidomskyy, and Qimiao Si. Strongly correlated materials. Advanced Materials, 24(36):4896-4923, 2012

40. Dieter Vollhardt. Dynamical mean-field theory for correlated electrons. Annalen der Physik, 524(1):1-19, 2012.

41. Hung T Dang, Xinyuan Ai, Andrew J Millis, and Chris A Marianetti. Density functional plus dynamical mean-field theory of the metal-insulator transition in early transition-metal oxides. Physical Review B, 90(12):125114, 2014

42. Lucas K Wagner. Transition metal oxides using quantum monte carlo. Journal of Physics: Condensed Matter, 19(34):343201, 2007.

43. IA Nekrasov, NS Pavlov, and MV Sadovskii. Consistent lda'+ dmft approach to the electronic structure of transition metal oxides: Charge transfer insulators and correlated metals. Journal of Experimental and Theoretical Physics, 116(4):620-634, 2013.

44. Shintaro Hoshino and Yoshio Kuramoto. Itinerant versus localized heavy-electron magnetism. Physical review letters, 111(2):026401, 2013.

45. Philipp Werner, Armin Comanac, Luca De'Medici, Matthias Troyer, and Andrew J Millis. Continuous-time solver for quantum impurity models. Physical Review Letters, 97(7):076405, 2006.
46. Lode Pollet, Kris Van Houcke, and Stefan MA Rombouts. Engineering local optimality in quantum monte carlo algorithms. Journal of Computational Physics, 225(2):2249-2266, 2007.

47. Bela Bauer, LD Carr, Hans Gerd Evertz, Adrian Feiguin, J Freire, S Fuchs, Lukas Gamper, Jan Gukelberger, E Gull, Siegfried Guertler, et al. The alps project release 2.0: open source software for strongly correlated systems. Journal of Statistical Mechanics: Theory and Experiment, 2011(05):P05001, 2011.

48. EY Loh Jr, JE Gubernatis, RT Scalettar, SR White, DJ Scalapino, and RL Sugar. Sign problem in the numerical simulation of manyelectron systems. Physical Review B, 41(13):9301, 1990.

49. ML Reinle-Schmitt, C Cancellieri, Danfeng Li, Denis Fontaine, M Medarde, E Pomjakushina, CW Schneider, Stefano Gariglio, $\mathrm{Ph}$ Ghosez, J-M Triscone, et al. Tunable conductivity threshold at polar oxide interfaces. Nature communications, 3(1):1-6, 2012.

50. Emanuel Gull, Philipp Werner, Olivier Parcollet, and Matthias Troyer. Continuous-time auxiliary-field monte carlo for quantum impurity models. EPL (Europhysics Letters), 82(5):57003, 2008.

51. IA Nekrasov, EE Kokorina, EZ Kuchinskii, MV Sadovskii, S Kasai, A Sekiyama, and S Suga. Arpes spectral functions and fermi surface for la $1.86 \mathrm{sr} 0.14$ cuo 4 compared with lda+ dmft+ $\sigma$ $\mathrm{k}$ calculations. Journal of Experimental and Theoretical Physics, 110(6):989-994, 2010.

52. Emanuel Gull. Continuous-time quantum Monte Carlo algorithms for fermions. PhD thesis, ETH Zurich, 2008.

53. Kristjan Haule. Quantum monte carlo impurity solver for cluster dynamical mean-field theory and electronic structure calculations with adjustable cluster base. Physical Review B, 75(15):155113, 2007.

54. Gabriel Kotliar, Sergej Y Savrasov, Kristjan Haule, Viktor S Oudovenko, O Parcollet, and CA Marianetti. Electronic structure calculations with dynamical mean-field theory. Reviews of Modern Physics, 78(3):865, 2006.

55. Steven R White. Density matrix formulation for quantum renormalization groups. Physical review letters, 69(19):2863, 1992.

56. H Makino, IH Inoue, MJ Rozenberg, F Iga, Y Aiura, and S Onari. Optical spectra of the correlated metallic system ca1- xsrxvo3. Physica B: Condensed Matter, 237:56-58, 1997.

57. Mauro Iazzi and Matthias Troyer. Efficient continuous-time quantum monte carlo algorithm for fermionic lattice models. Physical Review B, 91(24):241118, 2015.

58. Lewin Boehnke, Hartmut Hafermann, Michel Ferrero, Frank Lechermann, and Olivier Parcollet. Orthogonal polynomial representation of imaginary-time green's functions. Physical Review $B, 84(7): 075145,2011$

59. Alessandro Toschi, AA Katanin, and Karsten Held. Dynamical vertex approximation: A step beyond dynamical mean-field theory. Physical Review B, 75(4):045118, 2007.

60. Robert Peters and Thomas Pruschke. Half-filled hubbard model on a bethe lattice with next-nearest-neighbor hopping. Physical Review B, 79(4):045108, 2009.

61. Gabriel Kotliar, Sergej Y Savrasov, Gunnar Pálsson, and Giulio Biroli. Cellular dynamical mean field approach to strongly correlated systems. Physical review letters, 87(18):186401, 2001.

62. Satoshi Okamoto, Andrew J Millis, Hartmut Monien, and Andreas Fuhrmann. Fictive impurity models: An alternative formulation of the cluster dynamical mean-field method. Physical Review B, 68(19):195121, 2003.

63. Eva Pavarini, Erik Koch, Dieter Vollhardt, and Alexander Lichtenstein. Dmft at 25: Infinite dimensions: Lecture notes of the autumn school on correlated electrons 2014, volume 4. Forschungszentrum Jülich, 2014.

64. Michael Potthoff. Self-energy-functional approach to systems of correlated electrons. The European Physical Journal B-Condensed Matter and Complex Systems, 32(4):429-436, 2003.

65. Andreas Fuhrmann, Satoshi Okamoto, Hartmut Monien, and Andrew J Millis. Fictive-impurity approach to dynamical mean- 
field theory: A strong-coupling investigation. Physical Review B, 75(20):205118, 2007.

66. Robert O Jones and Olle Gunnarsson. The density functional formalism, its applications and prospects. Reviews of Modern Physics, 61(3):689, 1989.

67. Richard N Silver, Devinderjit S Sivia, and James E Gubernatis. Maximum-entropy method for analytic continuation of quantum monte carlo data. Physical Review B, 41(4):2380, 1990.

68. Mark Jarrell, Th Maier, C Huscroft, and S Moukouri. Quantum monte carlo algorithm for nonlocal corrections to the dynamical mean-field approximation. Physical Review B, 64(19):195130, 2001.

69. Gopi Chandra Kaphle, Shreemoyee Ganguly, Rudra Banerjee, Radheshyam Banerjee, Rabi Khanal, Chandra Mani Adhikari, Narayan Prasad Adhikari, and Abhijit Mookerjee. A study of magnetism in disordered pt-mn, pd-mn and ni-mn alloys: an augmented space recursion approach. Journal of Physics: Condensed Matter, 24(29):295501, 2012

70. P Blaha, K Schwarz, GK Madsen, D Kvasnicka, and J Luitz. Wien2k: An augmented plane wave plus local orbitals program for. Calculating Crystal Properties, Karlheinz Schwarz, Techn. Universitat Wien, 2001.

71. Durga Paudyal, VK Pecharsky, and KA Gschneidner Jr. Electronic structure, magnetic properties, and magnetostructural transformations in rare earth dialuminides. Journal of Applied Physics, 115(17):17E127, 2014.

72. Alyn DN James, Markus Aichhorn, and James Laverock. Quantum confinement induced metal-insulator transition in strongly correlated quantum wells of srvo 3 superlattices. Physical Review Research, 3(2):023149, 2021.

73. Anders W Sandvik. Constrained sampling method for analytic continuation. Physical Review E, 94(6):063308, 2016.

74. Evan Sheridan, Cédric Weber, Evgeny Plekhanov, and Christopher Rhodes. Continuous-time quantum monte carlo solver for dynamical mean field theory in the compact legendre representation. Physical Review B, 99(20):205156, 2019.

75. Emanuel Gull, Andrew J Millis, Alexander I Lichtenstein, Alexey N Rubtsov, Matthias Troyer, and Philipp Werner. Continuous-time monte carlo methods for quantum impurity models. Reviews of Modern Physics, 83(2):349, 2011.

76. O Gunnarsson, MW Haverkort, and G Sangiovanni. Analytical continuation of imaginary axis data for optical conductivity. Physical Review B, 82(16): 165125, 2010.

77. E Müller-Hartmann. Correlated fermions on a lattice in high dimensions. Zeitschrift für Physik B Condensed Matter, 74(4):507-512, 1989.

78. Anders W Sandvik and Juhani Kurkijärvi. Quantum monte carlo simulation method for spin systems. Physical Review B, 43(7):5950, 1991.

79. Walter Kohn and Lu Jeu Sham. Self-consistent equations including exchange and correlation effects. Physical review, 140(4A):A1133, 1965.

80. Eva Pavarini, Dieter Vollhardt, Erik Koch, and Alexander Lichtenstein. Dmft: From infinite dimensions to real materials. Technical report, Theoretische Nanoelektronik, 2018.

81. R Martin. Electronic structure-basic theory and practical methods, cambridge univ. Pr., West Nyack, NY, 2004.

82. MJ Veit, MK Chan, BJ Ramshaw, Rémi Arras, R Pentcheva, and Y Suzuki. Three-dimensional character of the fermi surface in ultrathin latio 3/srtio 3 heterostructures. Physical Review B, 99(11):115126, 2019.

83. KSD Beach, RJ Gooding, and F Marsiglio. Reliable padé analytical continuation method based on a high-accuracy symbolic computation algorithm. Physical Review B, 61(8):5147, 2000. 